\title{
Sharing Transnational Experiences of Psychogeriatric Units Dedicated to the Management of Behavioral and Psychological Symptoms in Demented Patients
}

\author{
Giuseppe Bellelli, MD*† and Marco Trabucchi, MD $\ddagger$
}

\begin{abstract}
T he occurrence of Behavioral and Psychological Symptoms of Dementia (BPSD) is one of the major problems associated with dementia, affecting not only the patient's outcomes but also the caregiver's health status and quality of life. ${ }^{1}$ Despite the progress in the knowledge of dementia, the management of BPSD continues to be a challenge for physicians and health workers in their everyday clinical practice. Indeed, nonpharmacological approaches are often insufficient to control the manifestation of BPSD if used alone. ${ }^{2}$ Nonetheless, studies demonstrated that the pharmacological treatment of BPSD with antipsychotic drugs is burdened by a high risk of adverse events, including extrapyramidal signs, falls, cerebrovascular events, and deaths, ${ }^{3}$ and, in this context, models of care attempting to combine nonpharmacological approaches with a careful use of drugs are obviously needed to improve assistance for people with BPSD.

Consistent with this approach, the French Government has recently developed a project, the "Plan Alzheimer" 2008 to 2012, in which newly designed intermediate-term care units, Cognitive and Behavioral Units (CBUs), have been created in addition to the traditional referral units, the so-called Acute Psychogeriatric Units (APUs), for the management of patients with BPSD. ${ }^{4}$ In a recent issue of Alzheimer disease and associated disorders, Koskas et $\mathrm{al}^{4}$ reported the results of a pilot study of CBUs, comparing data with those from APUs. The authors found that, in comparison with APUs, patients from CBUs are significantly more cognitively impaired and have more specific BPSD (ie, nighttime and aberrant motor behavior and agitation), while they are less impaired in nutritional and health status. The authors conclude that the 2 programs serve populations with different clinical needs, and, therefore, the 2 settings (ie, CBU and APU) do not act competitively. Another relevant finding is that, according to the physician's impression, the majority of patients served by either CBUs or APUs showed a marked improvement in their overall condition at discharge.

Despite these positive outcomes, however, the findings of the Koskas' study should be interpreted with caution. The small sample size and the lack of a control group among nonhospitalized patients with dementia limit their conclusions. Moreover, the authors do not address the crucial question of how CBUs and APUs might coordinate their activities in an integrated network of care for patients with dementia and their caregivers. Indeed, a health care delivery system is most efficient if it is able to fulfill its proper aims using all the available resources at a given condition. ${ }^{5}$ Therefore, the principle of efficiency requires that the system's resources be allocated in a synergistic manner to maximize this capacity. In the case of dementia services, this means that each unit of the network not only take care of patients with different clinical needs, but also that the sources of admission and the destinations at discharge from these units are not the same, but the efforts of the different programs remain coordinated. In fact, if sources of patient's admission and destination at discharge overlap within the 2 settings, the risk of duplicates significantly increases.

An Italian experience with the "Plan Alzheimer" of the Lombardia region (8 million inhabitants) addresses this issue of coordination and efficiency. This program was initiated in March of 1995 with the aim to improve the care of patients with dementia, ${ }^{6}$ promoting the creation of both Special Care Units (SCU), ${ }^{7-8}$ and Geriatric Evaluation and Rehabilitation Units for Dementia (GERU-D). ${ }^{9}$ In addition, in September 2000, 71 Alzheimer's Evaluation Units (AEUs) were created in the Lombardia region, as a part of a project financed by the
\end{abstract}

From the *Department of Clinical and Preventive Medicine, University of Milano-Bicocca and Geriatric Clinic, S. Gerardo Hospital, Monza; $\dagger$ Department of Rehabilitation and Aged Care, Ancelle della Carità Hospital, Cremona and Geriatric Research Group; and $\$$ Tor Vergata University, Rome and Geriatric Research Group, Brescia, Italy.

The authors declare no conflicts of interest.

Reprints: Giuseppe Bellelli, MD, Department of Clinical and Preventive Medicine, University of Milano-Bicocca and Geriatric Clinic, S. Gerardo Hospital, Monza, Italy (e-mails: giuseppe.bellelli@unimib.it; giuseppebellelli@libero.it).

Copyright (C 2011 by Lippincott Williams \& Wilkins 
Italian Minister of Health for the care of patients with dementia ("CRONOS project," www.Alzheimer-cronos. org). ${ }^{10}$ These units (ie, SCU, GERU-D, and AEUs) have different goals and different patient selection criteria, with different clinical settings. The SCUs are located in nursing homes and admit patients with severe dementia with BPSD who cannot return home. The GERU-D are located in rehabilitation facilities outside of hospitals and are designed for patients with dementia who may return home but have transient (moderate or severe) BPSD, not manageable at home, including delirium or any other problem requiring a close somatic assessment or emergency care. The AEUs are located in hospitals for out-patients who have dementia and stable clinical conditions, although they might require pharmacological treatments for BPSD.

In the Lombardia region, GERU-Ds are settings comparable to French CBUs. Data available from a study in this context ${ }^{9}$ suggest that GERU-Ds are capable of effectively addressing patients' clinical needs without causing extra burden to caregivers. Indeed, a high proportion of patients are directly admitted from home (80\%) and discharged to home $(78 \%)$ after a significant improvement of BPSD (Neuropsychiatric Inventory, average score, SD $23.1 \pm 16.5$ and $14.5 \pm 14.9$ on admission and at discharge, respectively). In addition, reduction of BPSD without increasing the use of neuroleptics or sedatives was a relevant finding from this study. Further research is, however, warranted to assess GERU-D effectiveness including a control group in a randomized design.

In conclusion, the data from the French and Italian experiences do not allow drawing inferences about the opportunity to incorporate these units into a care network for patients with dementia. Nonetheless, both these models attempt to provide comprehensive care for patients with severe BPSD and can therefore be considered as potential avenues for research and development to address this important health care issue.

\section{REFERENCES}

1. Sink KM, Holden KF, Yaffe K. Pharmacological treatment of neuropsychiatric symptoms of dementia: a review of the evidence. JAMA. 2005;293:596-608.

2. Lawlor BA. Behavioral and psychological symptoms in dementia: the role of atypical antipsychotics. J Clin Psychiatry. 2004;65(Suppl 11):5-10.

3. Jeste DV, Blazer D, Casey D, et al. ACNP White Paper: update on use of antipsychotic drugs in elderly persons with dementia. Neuropsychopharmachology. 2008;33:957-970.

4. Koskas P, Henry Feugeas MC, Saad S, et al. Management of behavioral and psychological symptoms of dementia in a dedicated psychogeriatric unit: a pilot experience. Alzheimer Dis Assoc Disord. 2011;25:184-186.

5. Black J. Efficiency. Dictionary of Economics. London: Oxford University Press; 2002.

6. Trabucchi M, Govoni S, Bianchetti A. Socio-economic aspects of Alzheimer's disease treatment. In: Giaconimi E, Becker R, eds. Alzheimer Disease: Therapeutic Strategies. Boston: Birkhäuser; 1994:459-463.

7. Bellelli G, Frisoni GB, Bianchetti A, et al. Special care units for demented patients: a multicenter study. Gerontologist. 1998;38: 456-462.

8. Nobili A, Piana I, Balossi L, et al. Alzheimer special care units compared with traditional nursing home for dementia care: are there differences at admission and in clinical outcomes? Alzheimer Dis Assoc Disord. 2008;22:352-361.

9. Ghianda D, Cornali C, Franzoni S, et al. Geriatric Evaluation and Rehabilitation Unit for Dementia: a model for the care of demented patients? G Gerontol. 2005;87-96.

10. Bellelli G, Lucchi E, Minicuci N, et al. Results of a multi-level therapeutic approach for Alzheimer's disease subjects in the "real world" (CRONOS project): a 36-week follow-up study. Aging Clin Exp Res. 2005;17:54-61. 